\title{
Lectin PCL inhibits the Warburg effect of PC3 cells by combining with EGFR and inhibiting HK2
}

\author{
HONG ZHANG ${ }^{1 *}$, XIA DU ${ }^{*}$, TING-TING SUN ${ }^{1}$, CHUN-LIU WANG ${ }^{1}$, YE LI $^{1}$ and SHOU-ZHEN WU ${ }^{2}$ \\ ${ }^{1}$ Institute of Traditional Chinese Medicine, Shaanxi Academy of Traditional Chinese Medicine, Xi'an, Shaanxi 710003; \\ ${ }^{2}$ Xi'an Children's Hospital, Xi'an, Shaanxi 710003, P.R. China
}

Received July 23, 2016; Accepted December 8, 2016

DOI: $10.3892 /$ or.2017.5367

\begin{abstract}
Prostatic carcinoma is the most aggressive tumor in adult men. Warburg effect is an important characteristic of tumor cell metabolism including prostate cancer cells, in which hexokinase 2 (HK2), a major rate-limiting enzyme involved in Warburg effect, is selectively upregulated. The lectin PCL is a mannose binding lectin which induces tumor cell apoptosis and autophagy. In the present study, we report that PCL could lower glucose consumption and lactate production, shift the Warburg effect by inhibiting the expression of HK2 in PC3 cells and the suppression of HK2 by siRNA reversed the effect of PCL on glucose consumption and lactate production. The expression of HK2 is closely related to epidermal growth factor receptor (EGFR) and downstream signaling pathway activation, therefore, we investigated the interaction of PCL with EGFR by western blot analysis and found that PCL could suppress the binding of epidermal growth factor (EGF) with EGFR and HK2 expression. Also, we explored the binding mechanism between the PCL and EGFR through molecular docking and molecular dynamics simulations and found that PCL bocked the active site of EGFR which is also the binding site of the nature ligand EGF, the resulting conformation has higher stability than EGF in complex with EGFR. The results indicated that PCL could competitively bind to EGFR binding pocket and then prevent EGF from binding to EGFR, blocking the autophosphorylation of the EGFR tyrosine kinase, after that the EGFR activation is inhibited. Collectively, our
\end{abstract}

Correspondence to: Professor Ye Li, Institute of Traditional Chinese Medicine, Shaanxi Academy of Traditional Chinese Medicine, Xihua Road, Xi'an, Shaanxi 710003, P.R. China

E-mail: liyelsj@163.com

Professor Shou-Zhen Wu, Xi'an Children's Hospital, Xi'an, Shaanxi 710003, P.R. China

E-mail:wushouzhen@qq.com

${ }^{*}$ Contributed equally

Key words: lectin, Warburg effect, hexokinase, epidermal growth factor receptor, molecular dynamics simulation studies concluded that PCL inhibits tumor cell glycolysis by combining with EGFR and reducing HK2 expression.

\section{Introduction}

Prostatic carcinoma is a metabolically active tumor which mostly occurs among elderly men. More than $80 \%$ of metastatic tumors achieve remission with androgen deprivation therapy, but develop hormone refractory prostate cancer leading to high mortality rates after 14-30 months of the median overall survival (1). Warburg effect is an important characteristic of tumor cell metabolism including prostate cancer cells, demonstrating that a tumor cell produces energy through glycolysis followed by pyruvic acid or lactic acid fermentation, rather than by tricarboxylic acid cycle or oxidative phosphorylation $(2,3)$. Hexokinase (HK), phosphofructokinase (PFK) and pyruvate kinase $(\mathrm{PK})$ are three major rate-limiting enzymes involved in Warburg effect (4-6), in which HK is the first enzyme in the regulation of glycolysis, promoting the production of fructose 6-phosphate from glucose. There are 4 subtypes (HK I-IV) in the HKs, which are relevant in tumor metastasis. HK2 protein is expressed in a high percentage of oncologists in human, such as lung, liver and prostate cancer (7-10). HK2 coordinates with voltage dependent anion channel (VDAC) on the mitochondrial outer membrane through hydrophobic N-terminal domain, which allows HK2 to utilize ATP produced by mitochondria and protect cells from being avoid of apoptosis (11). Likewise, EGFR/PI3K/Akt activation constitutes a hallmark of most cancer cells and plays an important role in tumor genesis and progression. The serine/threonine kinase (Akt) is a major downstream effector of phosphatidylinositol 3-kinase (PI3K) signaling pathway and a transducer of EGFR effects on cell survival (12), which constitutes a characteristic of most cancer cells and as such presumably plays important roles in Warburg effect of cancer cells. Akt-induced aerobic glycolysis may be mediated by multiple non-exclusive mechanisms, including the expression and membrane translocation of glucose transporters (GLUT) and effects on HK expression, activity and mitochondrial interaction (13-15).

The lectin PCL extracted from the Polygonatum sibiricum Red, is a galanthus nivalis agglutinin (GNA)-related lectin. Its molecular weight was estimated to be $4.2 \mathrm{kDa}$ by SDS-PAGE. The lectin could agglutinate rabbit erythrocytes to different degrees (13). Several studies have reported that 
PCL has antitumor activity, which is associated with multiple signal cascades $(14,15)$, but the precise mechanism of the lectin in inducing apoptosis remains to be clarified.

In the present study, we demonstrated that PCL could inhibit glucose consumption, lactate production and HK2 expression of human prostate cancer $\mathrm{PC} 3$ cells in vitro, thereby inducing PC3 cell metabolism. Because PCL has larger molecular structures that could target the outside of the cell, we hypothesized that PCL competed with epidermal growth factor (EGF) for binding to EGFR, suppressing activation of PI3K/Akt downstream pathways. Therefore, subsequent in vitro experiments were performed to investigate the interaction of PCL with EGFR by western blot analysis, molecular docking and molecular dynamics simulations. Taken together, these studies explored the intricate apoptosis-inducing mechanisms of PCL with various approaches.

\section{Materials and methods}

Materials and chemicals. The rhizome of Polygonatum sibiricum was collected from the Red River Valley located in the Taibai Mountain of China. Human prostate cancer cell lines (PC3) were obtained from the Institute of Biochemistry and Cell Biology (Shanghai, China). Anti-Hexokinase type $2\left(\mathrm{HK}_{2}\right)$, pyruvate kinase $\left(\mathrm{PKM}_{2}\right)$ and phosphofructokinase $(\mathrm{PFK})$ antibody were supplied by the Cell Signaling Technology, Inc. (Danvers, MA, USA). EGF was obtained from the Cell Signaling Technology. The X-tremeGENE transfection reagent was purchased from Roche Diagnostics Corp. (Indianapolis, IN, USA). Real-time PCR reagents and primer were purchased from Takara Shuzo Co., Ltd. (Kyoto, Japan). Chromatograph was supplied by General Electric Co. (Boston, MA, USA). HPLC instrument was purchased from Waters Co. (Milford, MA, USA). Real-time PCR was purchased from (Life Technologies (Carlsbad, CA, USA). HK2 interference fragment and reference were purchased from Shanghai GenePharma Co., Ltd. (Shanghai, China). The crystal structures of the extracellular domain of epidermal growth factor receptor (EGFR), the natural ligand epidermal growth factor (EGF) and the polygonatum cyrtonema lectin (PCL) obtained from RCSB Protein Data Bank were used as templates, PDB ID: 1NQL_A (16), 1NQL_B (16) and 3A0D_A (17). Protein-protein docking of the EGFR and PCL were carried out by using the online ClusPro 2.0 server (18-21). The binding structure of the EGFR with the natural ligand EGF was the crystal structure, PDB ID: 1NQL (16).

Extraction and purification of PCL. The fresh rhizome of Polygonatum sibiricum Red (100 g) was ground into small pieces and extracted with phosphate-buffered saline (PBS) at $-4^{\circ} \mathrm{C}$ for $12 \mathrm{~h}$ followed by centrifugation at $5120 \mathrm{x} \mathrm{g}$ for $15 \mathrm{~min}$. The supernatant was precipitated by saturated ammonium sulfate with a saturation degree of $80 \%$. After centrifugation, the precipitate was dissolved with $50 \mathrm{ml}$ PBS and dialyzed against PBS. The dialysate was subjected to chromatography on an S-100 molecular sieve column and eluted with Tris-HCI buffer of $\mathrm{pH} 7.0$, the eluent was loaded on a cation exchange column and eluted with PBS of $\mathrm{pH}$ 7.2. The final eluent was freeze-dried to give the PCL (22).
Glucose uptake assay. To measure glucose content, PC3 cells were plated into 6 -well plates $\left(1 \times 10^{5}\right.$ cells/well $)$ and treated with $50 \mu \mathrm{g} / \mathrm{ml} \mathrm{PCL}$ for $24 \mathrm{~h}$, then the cells were grown in Dulbecco's modidied Eagle's medium (DMEM) containing $10 \%$ fetal bovine serum (FBS) for $48 \mathrm{~h}$ at $37^{\circ} \mathrm{C}$ in an atmosphere of $5 \% \mathrm{CO}_{2}$. The supernatants were collected, the glucose contents were assayed by ELISA (23). The assay was repeated 3 times.

Lactate production assay. Lactate content was analyzed by ELISA according to the manufacturer's instructions (23). In brief, PC3 cells were added onto 6-well plates at a density of $1 \times 10^{5}$ cells/well and treated with $50 \mu \mathrm{g} / \mathrm{ml}$ PCL for $24 \mathrm{~h}$. The cultures were incubated at $37^{\circ} \mathrm{C}$ in an atmosphere of $5 \% \mathrm{CO}_{2}$ for $48 \mathrm{~h}$. The supernatants were collected and quantified by a microplate reader. The assay was repeated 3 times.

Western blot analysis. Western blot analysis was carried out as previously described (23). Cells were fully lysed in a cell lysis buffer to prepare protein extracts. The protein concentrations were determined using the BCA assay. Briefly, the proteins $(60 \mu \mathrm{g})$ extracted from cultured PC 3 cells by immunoprecipitation and immunoblotting with corresponding antibodies were loaded and separated by SDS polyacrylamide gel electrophoresis (SDS-PAGE) and transferred with $120 \mathrm{~V}$ constant voltage for $2 \mathrm{~h}$, then blocked in $5 \%$ bovine albumic (BSA) for $30 \mathrm{~min}$ at room temperature. The nitrocellulose membranes were incubated with primary antibody for $1 \mathrm{~h}$. The blots were washed with PBST buffer for 3 times (10 min/each time). Addition of a secondary antibody was conjugated to HRP and incubated for $30 \mathrm{~min}$. The cells were examined using ECL glow kits. The assay was repeated 3 times.

siRNA interference. The PC3 cells were grown in DMEM supplemented with $10 \%$ FBS and penicillin-streptomycin (1\%) and transfected after cell density to $80 \%$ according to the manusfacturer's instructions (24). siRNA-HK2 and siRNAEGFR were synthetized by Guangzhou Ruibo Biotechnology, Co. (Guangzhou, China).

Quantitative real-time PCR assay. The miRNA expression of HK2 in human prostate cancer PC3 cells was analyzed by real-time PCR using TaqMan MicroRNA (24). The cDNA was reverse transcribed from the total RNA extract and isolated from the PC3 cells treated with PCL using gene-specific primers according to the TaqMan MicroRNA assay protocol. Real-time PCR was performed using cDNA template. The reactions were incubated at $95^{\circ} \mathrm{C}$ for $2 \mathrm{~min}$, at $93^{\circ} \mathrm{C}$ for $40 \mathrm{sec}$, at $60^{\circ} \mathrm{C}$ for $35 \mathrm{sec}$ and at $72^{\circ} \mathrm{C}$ for $30 \mathrm{sec}$, respectively and cycled 40 times. The assay was run in intriplicate.

MTT assay. Cell viability was measured by the MTT assay according to the providers' instructions (Invitrogen, Carlsbad CA, USA) (24). In brief, PC3 cells were plated into 96-plates (5,000 cells/well). The PCL solution was added into each well. Cell viability was detected at $24 \mathrm{~h}$. Then, $100 \mu \mathrm{l}$ fresh medium and $10 \mu 1$ 3-(4,5-dimethylthiazol-2-yl)-2,5-diphenyltetrazolium bromide (MTT) were added to each well, and then the plate was incubated for an additional $4 \mathrm{~h}$ at $37^{\circ} \mathrm{C}$ in an atmosphere of $5 \% \mathrm{CO}_{2}$. The medium was then removed 

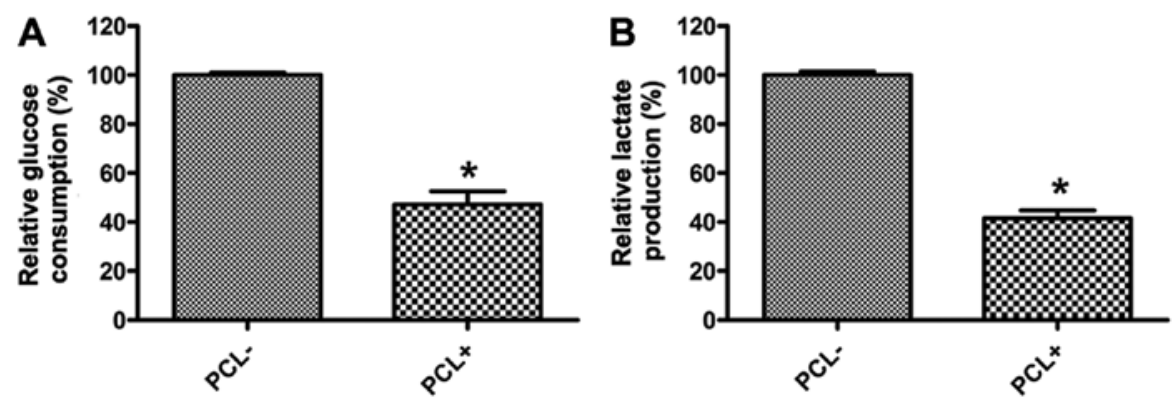

Figure 1. PCL inhibits glucose uptake and lactate production in prostate cancer PC 3 cells. (A) PC3 cells were plated at $80 \%$ confluence and treated with $50 \mu \mathrm{g} / \mathrm{ml}$ of PCL. Twenty-four hours later, the cells were given fresh media with $10 \%$ FBS and incubated for an additional $48 \mathrm{~h}$. The glucose uptake was evaluated by ELISA. (B) Cells were treated as in (A). Forty-eight hours later, the level of lactate production was determined by ELISA and all experiments were repeated 3 times.

and the crystals were solubilized with $100 \mu$ of dimethyl sulfoxide (DMSO) for $10 \mathrm{~min}$. The absorbance was measured at a wavelength of $490 \mathrm{~nm}$. Cell viability was normalized as relative percentages in comparison with the untreated control and the assay was repeated 3 times.

Molecular docking and molecular dynamics simulations. We introduced the well-tempered metadynamics simulation $(25,26)$ to NAMD $(27)$ in a portable plugin PLUMED (28) for exploring the binding mechanism between the PCL and EGFR through free-energy calculations. The temperature is $310 \mathrm{~K}$, and the value of bias-factor equal to 6.0 to allow for the system to escape from the initial local minimum in the time scale of this simulation. The metadynamic simulation starts as a standard MD simulation, but after 1 ps an artificial potential hill is added by using the PLUMED (28). After another $1 \mathrm{ps}$ a new hill is added and so forth. These potential hills accumulate and gradually disfavor the free energy minima having been already visited and thus facilitate an exploration of the whole free energy space. At the end of the simulation, the free energy surface is completely flooded and the system can walk on the free energy surface without barriers.

All the simulations were carried out under a constant pressure of 1 bar and a constant temperature of $310 \mathrm{~K}$. The minimization was based on the steepest descent method. The electrostatics potential and the van der Waals interactions were calculated with a non-bonded cutoff of $12 \AA$ based on the Particle Mesh Ewald (PME) method (29). The constant temperature and pressure were calculated by the langevin thermostat $(30,31)$ and langevin barostat method (32), respectively. All the MD simulations were performed with a time step of $1 \mathrm{fs}$. The trajectories of MD simulations were stored every $5 \mathrm{ps}$ for the analysis of MD simulated data, and $10 \mathrm{~ns}$ MD simulations were performed on the systems. All the MD simulations were carried out using NAMD (27) (version 2.9) with the AMBER force field (33) and explicit TIP3P water.

Statistical analysis. The results are expressed as the means \pm standard deviation, and analyzed by using SPSS 17.0 software. Statistical significance was compared with other groups for significance by one-way ANOVA followed by Bonferroni post hoc test (multiple comparison tests) using GraphPad Prism version 5.01. $\mathrm{P}<0.05$ was considered statistically significant.

\section{Results}

PCL inhibits the Warburg effect of prostate cancer PC3 cells. A previous study revealed that it is common in tumor that glucose uptake and lactate production increase through nonexclusive dependent signaling cascade (34). Therefore, to study whether PCL is involved in the Warburg effect, we analyzed the effect of PCL on PC3 metabolism. The results indicated that PCL inhibited PC3 cells glucose absorption obviously (Fig. 1A), and it also reduced the lactate production (Fig. 1B).

The effect of PCL on expression of HK2, PFK or PKM2 in $P C 3$ cells. It was earlier identified that HK2, PFK and PKM2 are expressed in a high percentage of many tumors (3-5). In the present study, we explored the expression of the three key rate-limiting enzymes in PC3 cells treated with PCL. The results of western blot analysis and real-time PCR indicated that PCL enhanced the expression of HK2 and had no effects on the expression of PFK or PKM2 in PC3 cells (Fig. 2A and B). However, with siRNA-HK2 interference, the effect of PCL on glucose absorption and lactate production in PC3 cells was reversed (Fig. 2C and D). The results showed that PC3 cells exhibited a comparable respose to PCL treatment, PCL could inhibit tumor cell glycolysis through reducing HK2 expression.

PCL inhibits the cell viability of prostate cancer PC3 cells. Reduced levels of HK2 in PCL-treated PC3 cells prompted us to further study the effect of PCL on the cell viability of prostate cancer PC 3 cells. Our western blot analysis conducted on HK2 expression showed that PCL inhibited the activity of PC 3 cells, after siRNA-HK2 interference, and had no effect on the activity of PC3 cells (Fig. 3).

PCL reduces $H K 2$ expression by competitively binding to EGFR binding pocket with EGF and causes accelerated apoptosis of the tumor cells. EGFR/PI3K/Akt signaling pathway activation upregulated the expression of HK2. PCL has larger molecular structures that could target the outside of the cell, we hypothesized that PCL could compete with EGF for binding to EGFR, suppress activation of PI3K/Akt downstream pathways and HK2 expression. As shown in Fig. 4, introduction of the EGF significantly increased the level of HK2 in PC3 cells. With PCL treatment, the expression of HK2 was suppressed. 

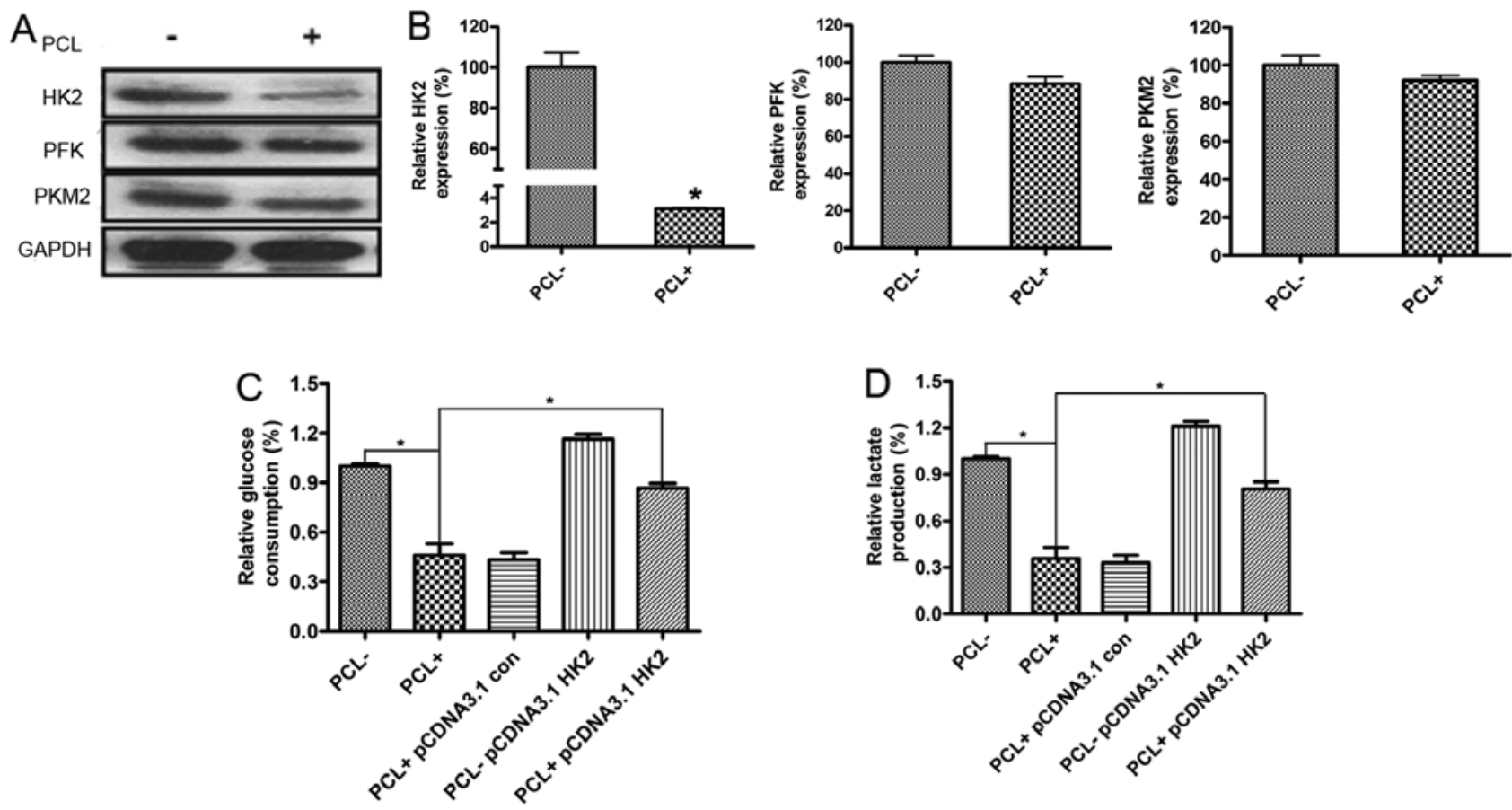

Figure 2. PCL inhibits the expression of HK2 in prostate cancer PC3 cells. (A) PC3 cells were plated at $80 \%$ confluence and treated with $50 \mu \mathrm{g} / \mathrm{ml} \mathrm{PCL}$ for $24 \mathrm{~h}$. The cells were evaluated for the expression of HK2, PFK and PKM2 by western blot analysis. (B) Cells were treated as in (A). Twenty-four hours later, the levels of HK2, PFK and PKM2 were determined by real-time PCR. (C) PC3 cells were plated at $80 \%$ confluence and then transfected with the indicated plasmid vectors. Twenty-four hours later, the cells were given fresh media with 10\% FBS and incubated with $50 \mu \mathrm{g} / \mathrm{ml} \mathrm{PCL}$ for an additional $24 \mathrm{~h}$. The glucose uptake was evaluated by ELISA. The parental vector was pcDNA 3.1, which was used as an empty vector control. (D) Cells were prepared as in (C). Twentyfour hours later, the level of lactate production was determined by ELISA. All experiments were repeated 3 times.
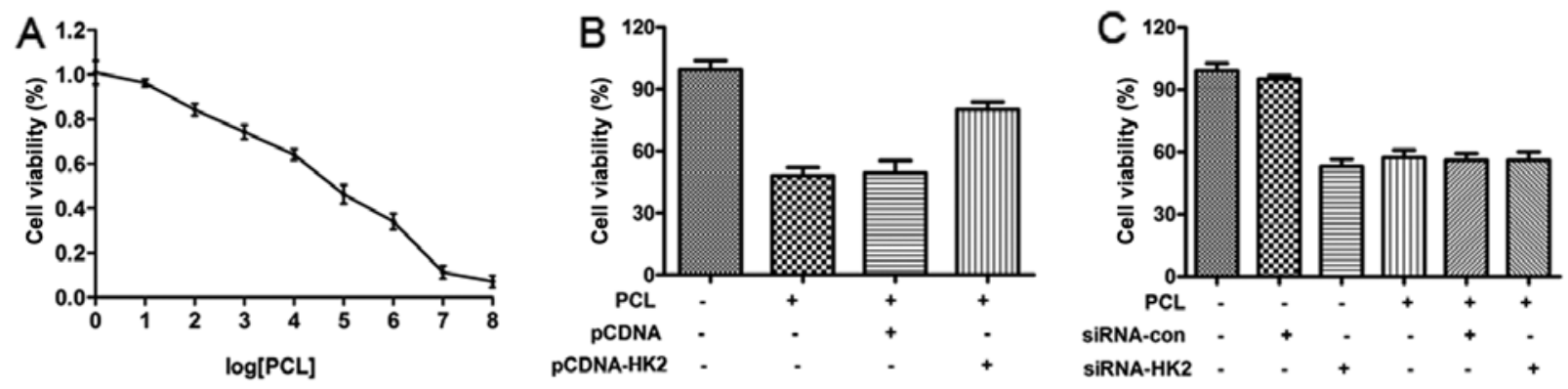

Figure 3. PCL inhibits the cell viability of prostate cancer PC3 cells. (A) PC3 cells were plated at $80 \%$ confluence and $0,6.25,12.5,25,50,100,200,400$ and $800 \mu \mathrm{g} / \mathrm{ml}$ PCL was added into each well, respectively. At $24 \mathrm{~h}$, the cell viability was detected by the MTT method. (B) PC 3 cells were plated at $80 \%$ confluence and $50 \mu \mathrm{g} / \mathrm{ml}$ PCL was added into each well. At $24 \mathrm{~h}$, the cell viability was detected by the MTT method. (C) Cells were plated at $80 \%$ confluence and transfected with siRNAs for HK2. After post-transfection, the cells were treated with $50 \mu \mathrm{g} / \mathrm{ml}$ PCL. At $24 \mathrm{~h}$, the cell viability was detected by the MTT method. All experiments were repeated 3 times.

These data supported the hypothesis that PCL could suppress the aerobic glycolysis of PC3 cells by combining with EGFR. To further verify the mechanism, we explored the binding mechanism between the PCL and EGFR through molecular dynamics simulations. The calculation of the free energy of binding from a two-dimensional potential of mean force (PMF) (35) along a reaction coordinate was completed using metadynamic method by NAMD (28) and PLUMED (28). It is because both EGF and PCL only go through the dual (X and $\mathrm{Y}$ ) directions out of the receptor control, the distance between the mass center of ligands and that of EGFR along these two directions was defined as the relevant reaction coordinate. Fig. 5 showed the PMF as a function of the distances between different ligands and receptors along the reaction coordinates within $10 \mathrm{~ns}$ metadynamics simulations. From the figure, it is clear that the lowest energy well is located in the pockets of the acceptor protein. The PMFs of different ligands have only one domain region, and the domain of PCL is larger than that of EGF. The hydrogen bond (HB) analysis provided evidence of benefits for this conclusion (Fig. 6). The numbers of $\mathrm{HB}$ between the PCL and protein are more than any other. There are only four HB between the EGF and EGFR, but fourteen for PCL. Due to the influence of HB, the binding of PCL to the receptor is more stable. Outside the combination region, the values of PMF present a trend of fluctuation. This may be the effect of the reservoir, to be more exact, ligands also can produce $\mathrm{HB}$ with the aquatic, and all that instantaneously and unsterdily. 


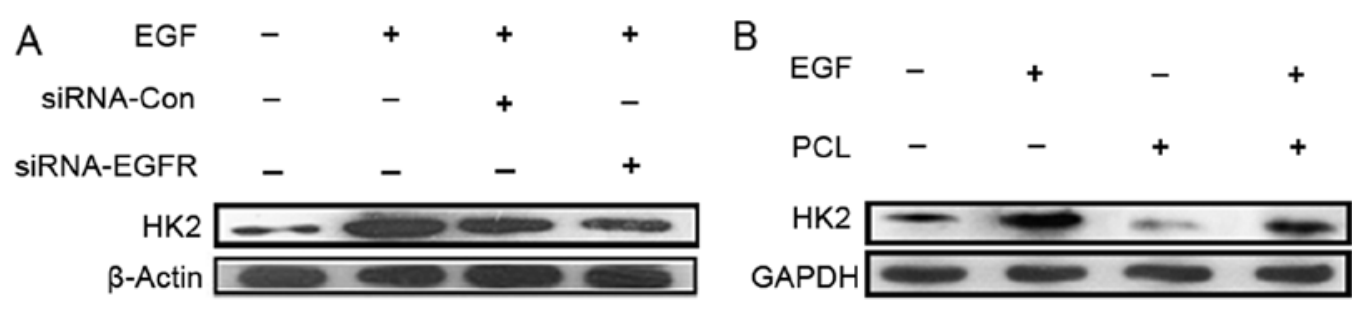

Figure 4. PCL competes with EGF for ligand binding in the extracellular domain of EGFR. (A) PC3 cells were plated at $80 \%$ confluence. The cells were treated with EGF, EGF and siRNA-EGFR respectively for $24 \mathrm{~h}$. The GAPDH was used as a reference gene. Control cells shown were treated with the reagent. Twenty-four hours later, the cells were given fresh media with 10\% FBS and incubated for an additional $24 \mathrm{~h}$. The cells were evaluated for the expression of HK2 by western blot analysis. (B) PC3 cells were plated at $80 \%$ confluence and treated with EGF, PCL, EGF and PCL respectively for $24 \mathrm{~h}$. The cells were treated as in (A). All experiments were repeated 3 times.
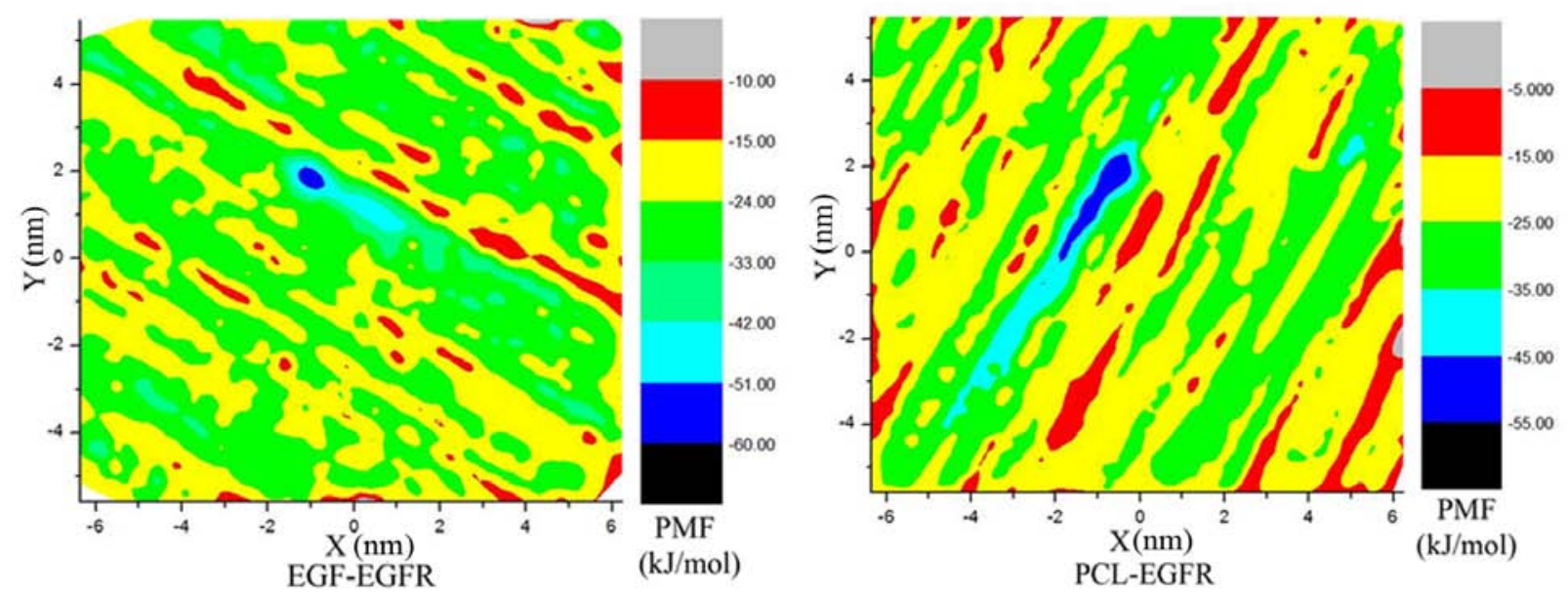

Figure 5. The PMF along the reaction coordinate within $10 \mathrm{~ns}$ simulations, and the reaction coordinate is defined as the distances between the mass center of the different ligands and the EGFR along the dual (X and Y) directions.

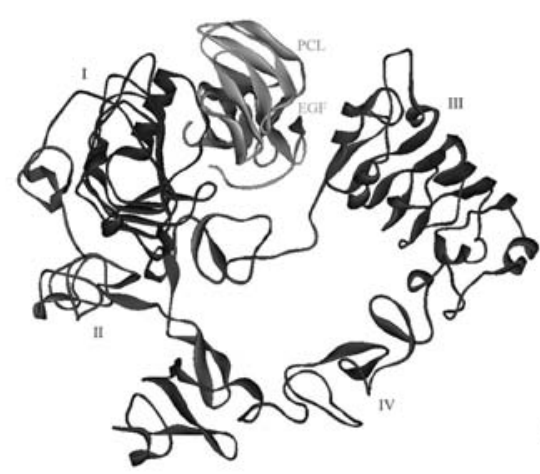

EGF/PCL-EGFR

A

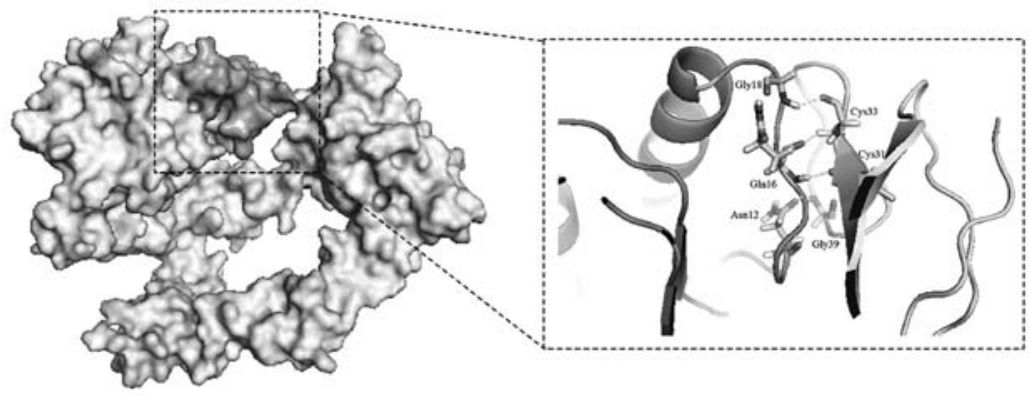

EGF-EGFR

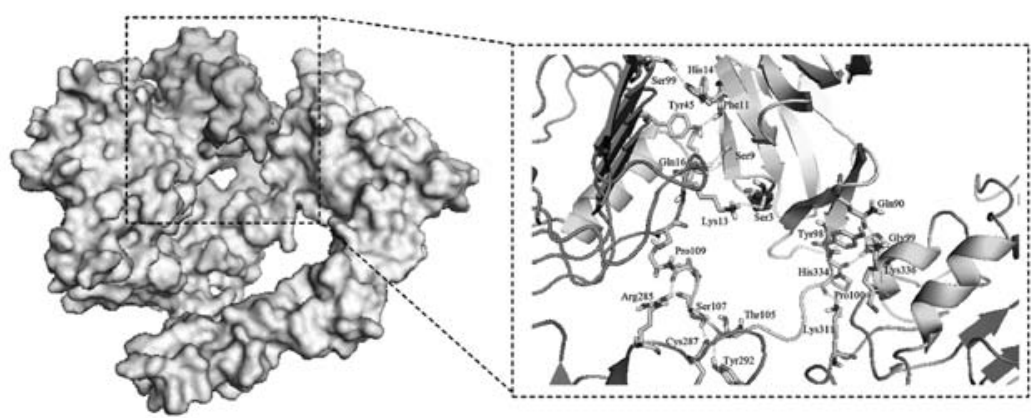

PCL-EGFR

B

Figure 6. The graphs of (A) docking results of the ligand EGF and PCL with the extracellular domain of EGFR; (B) the hydrophobic contacts and the hydrogen bond (HB) generated between EGFR and EGF or PCL. The dash lines represent H-bonds. 


\section{Discussion}

In the core high necrotic areas in any given tumor, the amount of oxygen observed is extremely low, thereby accelerating the cells to synthesize and obtain energy by glycolysis, which is termed the Warburg effect $(1,2)$. PCL is a mannose binding lectin. Previous studies have reported that PCL could induce tumor cell apoptosis and autophagy. Zhang and colleagues (13) found that PCL could induce apoptosis of L929 cell to murine fibrosarcoma in a caspase-dependent manner, as well as amplify TNF-induced L929 cell apoptosis. Liu and colleagues $(14,15)$ found that PCL treatment abrogated glutathione antioxidant system and induced mitochondria to generate ROS accumulation, resulting in p38-p53 activation. They also confirmed that the ROS-p38-p53 pathway was involved in PCL-induced autophagy. In view of these findings, we hypothesized that PCL might interact energy metabolism in cancer cells. Our findings therefore emphasized the importance of studying the effect of PCL on the Warburg effect of PC3 cells. To begin addressing this question, we investigated the effect of PCL on glucose consumption of PC 3 cells, a characteristic of the Warburg effect and found that the level of glucose upstake was strongly correlated to PCL treatment. Another characteristic of the Warburg effect is the reduction of pyruvate, the end product of glycolysis, to lactic acid, which is accompanied by the oxidation of NADH to $\mathrm{NAD}^{+}$. This reaction takes the place of the conversion of pyruvate to acetyl-CoA, which may enter the tricarboxylic acid (TCA) cycle (25). We next examined whether PCL treatment suppressed lactate production in PC3 cells and found that PCL could inhibit PC3 cells from producing lactate acid. These data indicated that PCL could suppress the Warburg effect of PC 3 cells.

The hallmark of the Warburg effect is the overexpression of several important rate-limiting enzymes, for example HK, PFK and PK. HK, involved in the first regulatory step, appears predominantly in an HK2 isoenzyme form in tumors which is bound to the mitochondrial outer membrane facing the cytosol. Microlocation of this enzyme enables preferential access to newly synthesized ATP for phosphorylating glucose, and it is resistant to product inhibition (36). Accumulated evidence suggests that codeletion of the tumor suppressor genes PTEN and p53 plays a crucial role in the development of castration-resistant prostate cancer in vivo. Wang and colleagues (37) demonstrated that HK2 is selectively upregulated by the combined loss of Pten and p53 in prostate cancer cells. Mechanistically, PTEN deletion increases HK2 mRNA translation through the activation of the AKT-mTORC14EBP1 axis, and p53 loss enhances HK2 mRNA stability through the inhibition of miR143 biogenesis. HK2-mediated aerobic glycolysis is required for Pten-/p53-deficiency-driven tumor growth in xenograft mouse models of prostate cancer. PFK, the 'gatekeeper' of glycolysis, catalyses the committed step of the glycolytic pathway by converting fructose-6-phosphate to ructose-1,6-bisphosphate, which is proposed to have important roles in metabolic reprogramming in cancer (36). $\mathrm{PKM}_{2}$ is a limiting glycolytic enzyme that catalyzes the final step in glycolysis, which is the key in tumor metabolism and growth (38). We therefore explored the expression of the three key rate-limiting enzymes in PC3 cells treated with PCL and found that PCL enhanced the expression of HK2 in PC3 cells, whereas, PCL has no effect on the expression of PFK or $\mathrm{PKM}_{2}$. The results showed that PC3 cells exhibited a comparable respose to PCL treatment, PCL inhibited tumor cell glycolysis through reducing HK2 expression. Futhermore, we examined the effect of suppressing the HK2 activity on the glucose upstake and lacate production in the PC 3 cells. The results showed that the upstake of glucose and the production of lactate were dramatically increased when the dominant negative HK2 mutants were introduced. In addition, we examined the effect of PCL on the PC 3 cell viability. The data showed that PCL inhibited the viability of PC3 cells, but after transfection with siRNA-HK2, it has no effect on the activity of PC3 cells. The results showed that PC 3 cells exhibited a comparable respose to PCL treatment, PCL was able to inhibit tumor cell glycolysis through reducing HK2 expression.

PCL has larger molecular structures $(4.2 \mathrm{kDa})$ that may target the outside of the cell (13). While EGFR is a widespread oncogenic signature in prostatic carcinoma, Akt (the serine/ threonine kinase) is a major downstream effector of EGFR/ PI3K that may induce aerobic glycolysis and increase HK2 expression in cancer cells through multiple non-exclusive mechanisms (15-17). Therefore, we hypothesized that PCL may combine with EGFR, suppress activation of PI3K/Akt downstream pathways and expression of HK2. Western blot analysis showed that the activation of EGFR significantly increased the expression of HK2 in PC 3 cells, but PCL treatment might inhibit this effect. These data support the hypothesis that PCL could suppress the aerobic glycolysis of PC3 cells by combining with EGFR. The EGFR consists of an extracellular ligand-binding domain, a single hydrophobic transmembrane region, and the intracellular part harbouring the highly conserved tyrosine kinase domain. Ligand binding induces the formation of homo- or heterodimers which subsequently trigger autophosphorylation of cytoplasmic tyrosine residues. These phosphorylated amino acids represent docking sites for a variety of signal transducers which regulate membrane-proximal steps of a complex signaling network ultimately defining the biological response to a given signal. The extracellular ligand-binding region has four domains (I-IV; Fig. 6). By the protein-protein docking and MD, we found that PCL can bind to the pocket between domain I and III, which is also the binding site of the nature ligand EGF. In short, PCL could competitively bind to EGFR binding pocket and then prevent EGF from binding to EGFR, block the autophosphorylation of the EGFR tyrosine kinase, after that the EGFR activation is inhibited. As a result, the PCL could inhibit the cell cycle progression, and cause accelerated apoptosis of the tumor cells.

Our current findings revealed that the plant lectin PCL treatment reduced PC3 cell growth, highlighting the distinct functions of PCL relative to tumor development. The illustrated mitochondrial function of PCL and its interaction with EGFR provided important insights into prostate tumor progression and its role in regulating mechanisms for tumor glycolysis. In summary, this study suggests that PCL may serve as a novel therapeutic drug in treating prostate cancer with EGFRmediated energy mechanisms. In future, the mechanisms of PCL and the downregulation of HK2 expression by EGFR mediation need to be addressed. 


\section{Acknowledgements}

The present study was supported by the Foundation of Science and Technology Resource of Shaanxi Province (no. 2016KTCL03-14).

\section{References}

1. Mahjoubi M, Azab M, Ghosn M, Theodore C and Droz JP: Phase II trial of ifosfamide in the treatment of metastatic hormonerefractory patients with prostatic cancer. Cancer Invest 8 : 477-481, 1990

2. Kroemer G and Pouyssegur J: Tumor cell metabolism: cancer 'Achilles' heel. Cancer Cell 13: 472-482, 2008.

3. Warburg O, Posener K and Negelein E: Ueber den stoffwechse dercarcinomzelle. Biochem Z 152: 309-344, 1924.

4. Ferlay J, Shin HR, Bray F, Forman D, Mathers C and Parkin DM Estimates of worldwide burden of cancer in 2008: GLOBOCAN 2008. Int J Cancer 127: 2893-2917, 2010.

5. Mayevsky A: Mitochondrial function and energy metabolism in cancer cells: past overview and future perspectives. Mitochondrion 9: 165-179, 2009.

6. Hanahan D and Weinberg RA: Hallmarks of cancer: the next generation. Cell 144: 646-674, 2011.

7. Jiang XZ, Yan LN, Sun GY, Zhao SY, Gu JM, Lei KJ, Zhu Y and Wang CS: Expression of hypoxia-inducible factor-1 alpha, hexokinase-II and lactate dehydrogenase-V in hepatocellular carcinoma and their biological significance. Chin J Cancer Prev Treat 1: 41-44, 2013.

8. Fan YX, Shi WM, Li J, Yin JL, Yang CH, Huang KL, Liu QZ, $\mathrm{Li} \mathrm{KB}$ and Wu JZ: Relationship between fluorodeoxyglucose uptake and overexpression of glucose transport protein 1 and hexokinase-II in early-stage nasopharyngeal carcinoma. Chin J Nucl Medi 30: 166-169, 2010.

9. Herrala AM, Porvari KS, Kyllönen AP and Vihko PT: Comparison of human prostate specific glandular kallikrein 2 and prostate specific antigen gene expression in prostate with gene amplification and overexpression of prostate specific glandular kallikrein 2 in tumor tissue. Cancer 92: 2975-2984, 2001.

10. He HC, Bi XC, Zheng ZW, Dai QS, Han ZD, Liang YX, Ye YK, Zeng GH, Zhu G and Zhong WD: Real-time quantitative RT-PCR assessment of PIM-1 and HK2 mRNA expression in benign prostate hyper-plasia and prostate cancer. Med Oncol 26 303-308, 2009

11. Pedersen PL: Warburg, me and Hexokinase 2: Multiple discoveries of key molecular events underlying one of cancers' most common phenotypes, the 'Warburg effect', i.e., elevated glycolysis in the presence of oxygen. J Bioenerg Biomember 39 211-222, 2007.

12. Bhaskar PT and Hay N: The two TORCs and Akt. Dev Cell 12: 487-502, 2007.

13. Zhang ZT, Peng H, Li CY, Liu JJ, Zhou TT, Yan YF, Li Y and Bao JK: Polygonatum cyrtonema lectin induces murine fibrosarcoma L929 cell apoptosis via a caspasedependent pathway as compared to ophiopogon japonicus lectin. Phytomedicine 18 : 25-31, 2010.

14. Liu B, Wu JM, Li J, Liu JJ, Li WW, Li CY, Xu HL and Bao JK: Polygonatum cyrtonema lectin induces murine fibrosarcoma L929 cell apoptosis and autophagy via blocking Ras-Raf and PI3K-Akt signaling pathways. Biochimie 92: 1934-1938, 2010.

15. Liu B, Cheng Y, Zhang B, Bian HJ and Bao JK: Polygonatum cyrtonema lectin induces apoptosis and autophagy in human melanoma A375 cells through a mitochondria-mediated ROS-p38-p53 pathway. Cancer Lett 275: 54-60, 2009.

16. Ferguson KM, Berger MB, Mendrola JM, Cho HS, Leahy DJ and Lemmon MA: EGF activates its receptor by removing interactions that auto-inhibit ectodomain dimerization. Mol Cell 11: 507-517, 2003

17. Ding JJ, Bao JK, Zhu DY, Zhang Y and Wang DC: Crystal structures of a novel anti-HIV mannose-binding lectin from Polygonatum cyrtonema Hua with unique ligand-binding property and super-structure. J Struct Biol 171: 309-317, 2010.
18. Kozakov D, Beglov D, Bohnuud T, Mottarella SE, Xia B, Hall DR and Vajda S: How good is automated protein docking? Proteins 81: 2159-2166, 2013.

19. Kozakov D, Brenke R, Comeau SR and Vajda S: PIPER: An FFT-based protein docking program with pairwise potentials. Proteins 65: 392-406, 2006.

20. Comeau SR, Gatchell DW, Vajda S and Camacho CJ: ClusPro: an automated docking and discrimination method for the prediction of protein complexes. Bioinformatics 20: 45-50, 2004.

21. Comeau SR, Gatchell DW, Vajda S and Camacho CJ: ClusPro: a fully automated algorithm for protein-protein docking. Nucl Acids Res 32: W96-W99, 2004.

22. Bao JK, Zeng ZK and Zhou H: Purification and characterization of the polygonatum cyrtonema Hua. Lectin II. Chin Biochem J 12: 165-170, 1996.

23. Velpula KK, Bhasin A, Asuthkar S and Tsung AJ: Combined targeting of PDKi and EGFR triggers regression of glioblastoma by reversing the Warburg effect. Cancer Res 73: 7277-7289, 2013.

24. Toschi A, Lee E, Thompson S, Gadir N, Yellen P, Michael drain C, Ohh M and Foster DA: Phospholipase D-mTOR requirement for the Warburg effect in human cancer cells. Cancer Lett 299: 72-79, 2010.

25. Barducci A, Bussi G and Parrinello M: Well-tempered metadynamics: A smoothly converging and tunable free-energy method. Phys Rev Lett 100:020603, 2008.

26. Ilott AJ, Palucha S, Hodgkinson P and Wilson MR: Well-tempered metadynamics as a tool for characterizing multicomponent, crystalline molecular machines. J Phys Chem B 117: 12286-12295, 2013.

27. Phillips JC, Braun R, Wang W, Gumbart J, Tajkhorshid E, Villa E, Chipot C, Skeel RD, Kalé L and Schulten K: Scalable molecular dynamics with NAMD. J Comput Chem 26: 1781-1802, 2005

28. Bonomi M, Branduardi D, Bussi G, Camilloni C, Provasi D, Raiteri P, Donadio D, Marinelli F, Pietrucci F, Broglia RA and Parrinello M: Plumed: A portable plugin for free-energy calculations with molecular dynamics. Comput Phys Commun 180: 1961-1972, 2009.

29. Darden T, York D and Pedersen L: Particle mesh Ewald: An $\mathrm{N} \cdot \log (\mathrm{N})$ method for Ewald sums in large systems. J Chem Phys 98: 10089-10092, 1993.

30. Adelman SA and Doll JD: Generalized langevin equation approach for atom/solid-surface scattering: general formulation for classical scattering off harmonic solids. J Chem Phys 64: 2375-2388, 1976.

31. Davidchack RL, Handel R and Tretyakov MV: Langevin thermostat for rigid body dynamics. J Chem Phys 130: 234101, 2009.

32. Feller SE, Zhang Y, Pastor RW and Brooks BR: Constant pressure molecular dynamics simulation: the Langevin piston method. J Chem Phys 103: 4613-4621, 1995.

33. Cornell WD, Cleplak P and Bayly CI: A second generation force field for the simulation of proteins, nucleic acids, and organic molecules. J Am Chem Soc 117: 5179-5197, 1995.

34. Liu B, Cheng Y, Bian HJ and Bao JK: Molecular mechanisms of Polygonatum cyrtonema lectin-induced apoptosis and autophagy in cancer cells. Autophagy 5: 253-255, 2009.

35. Kirkwood JG: Statistical mechanics of fluid mixtures. J Chem Phys 3: 300-313, 1935.

36. Mathupala SP, Ko YH and Pedersen PL: Hexokinase-2 bound to mitochondria: cancer's stygian link to the 'Warburg Effect' and a pivotal target for effective therapy. Semin Cancer Biol 19: 17-24, 2009.

37. Wang L, Xiong H, Wu F, Zhang Y, Wang J, Zhao L, Guo X, Chang LJ, Zhang Y, You MJ, et al: Hexokinase 2-mediated Warburg effect is required for PTEN- and p53-deficiency-driven prostate cancer growth. Cell Rep 8: 1461-1474, 2014.

38. Dong G, Mao Q, Xia W, Xu Y, Wang J, Xu L and Jiang F: PKM2 and cancer: The function of PKM2 beyond glycolysis. Oncol Lett 11: 1980-1986, 2016. 